\title{
Nano based therapy for biofilm management: mini review
}

\begin{abstract}
High toxicity, low host tolerance, narrow spectrum of antifungal drugs and increasing incidence of azole-resistance complicates treatment of invasive Candida infections. Due to the emergence of antimicrobial resistance as an increasing threat to global health care, arises the ne

ed to develop new and effective broad spectrum active antifungal agents. Thus understanding and combating drug resistance in Candida albicans is a toughest challenge being faced today and it's immensely important to develop an alternative coating that can inhibit biofilm formation on medical devices as implants and catheters. Additionally, the fascinating properties of nanomaterials and biocompatibility promises great potential in nanomedical applications, especially in medical microbiology. With appropriate modification of these synthetic materials, several disease infections can be cured and results have shown the use of these nanomaterials as diagnostic and immunotherapauetic agents. Since, antimicrobial resistance is becoming a major apprehension worldwide, nanomedicines offer an intriguing and promising solution for combating these high resistance pathogens by appropriate development of therauptic and novel diagnosis approaches. This mini review presents the current state of art in this emerging field.
\end{abstract}

Keywords: Biofilm, Candida albicans, Antifungal agents, Quantum dots, Quorum sensing
Volume 5 Issue 5 - 2017

\author{
Eepsita Priyadarshini,' Kamla Rawat,, ${ }^{2,3}$ \\ Bohidar HB',2 \\ 'School of Physical Sciences, Jawaharlal Nehru University, India \\ ${ }^{2}$ Special Centre for Nano Sciences, Jawaharlal Nehru University, \\ India \\ ${ }^{3}$ Inter University Accelerator Centre, India
}

\section{Correspondence: Kamla Rawat, Special Centre for Nano Sciences, Inter University Accelerator Centre, Jawaharlal Nehru University, India; Email kamla.jnu@gmail.com}

Co-correspondence: Bohidar HB, School of Physical Sciences, Special Centre for Nano Sciences, Jawaharlal Nehru University, India Email bohi0700@mail.jnu.ac.in

Received: May 24, 2017 | Published: July 05, 2017

\section{Introduction}

Biofilm represents an adherent community of microbial cells entrapped structurally in a polymeric matrix, communicating through the phenomenon of quorum sensing. Quorum sensing is the physiological mechanism of cellular communication used by microbial cells involving the release, sensing and response to signaling molecules called as autoinducers (AIs) . ${ }^{1}$ It basically involves the polymeric matrix made of polysaccharide secreted by the microbial cells that makes the microbes sessile and resistant to antimicrobial therapy . ${ }^{2}$ Biofilms mostly develop on surface of medical devices and inert surfaces, which has become a major concern. Figure 1a shows typical example of mechanism of $P$. aeruginosabiofilm formation, where the flagella and type IV pilli assist in providing mobility, and via quorum sensing maturation of the biofilm structure occurs. Figure $1 \mathrm{~b}$ shows two different pathways by which biofilms detach from the surface. One of the mechanisms involves the hydrolysis of the polysaccharide matrix and the release of the motile planktonic cells. The other means occurs by the physical process of fragmentation of the microcolony and subsequent detachment.$^{3}$

In this review, we have presented the current state of art of biofilm formation and the role of various antifungal agents in their inhibition. As biofilm formation on medical devices is a major concern and a number of microbes (bacteria and fungus) are known to cause infection and biofilm. Their irradiation by the conventional antifungal therapy is becoming difficult because of multi-drug resistance. Therefore, we present a brief discussion of the usefulness of the new emerging nanomaterials (functionalized QDs) in this regards.

\section{Discussion}

Amongst the biofilms producing microbes, Candida albicans is a well-studied model organism found as an opportunistic pathogen in humans. It is responsible for a number of infections like invasive candidiasis, a primary cause of mortality in immune compromised patients ${ }^{4}$ C. albicans form bio films by organizing themselves in yeast and hyphae form in an extracellular matrix and hence offer a high resistance to antifungal treatments.$^{5}$ Furthermore, Candidashows high resistance to antifungal therapy resulting in failure of treatments and recalcitrant infection along with the development of multi-drug resistant mutans.$^{6}$ Azole resistant clinical isolates of $C$. albicans acquire crossresistance to many unrelated drugs, a phenomenon termed as MultiDrug Resistance (MDR), which is a manifestation of multifactorial phenomenon with various mechanisms known to contribute towards its development. Over expression of drug efflux pumps belonging to the ABC superfamily of transporters e.g. Candida drug resistance protein (CDR1 and CDR2) and MFS transporters e.g. MDR1, FLU1, BENr; molecular alterations of the lanosterol $14-\alpha$ demethylase, the target enzyme of the azoles being the basic two mechanisms. A wide range of antifungal agents as antibiotics, drugs, quaternary ammonium compounds have been used to treat such infections, however these are associated with major disadvantages of antibiotic resistance, limited activity, environmental toxicity and complex synthesis procedure ${ }^{7}$

The potential of nanoparticles and their conjugates in medicine has been investigated for early detection, diagnosis and disease treatment. In this regard, researchers focused on the use of novel nanomaterials of silver, metal oxides, carbon nanotubes and graphene as antifungal agents. "Nano based therapy" provides promising approach for management of infection and biofilm formation. Alas, there are also specific shortcomings associated, as in graphene induces apoptosis in mammalian cells due to high oxidative stress while carbon nanotubes are toxic to microbial as well as human cells ${ }^{8}$ Nanosilver (AgNPs) has also been greatly investigated for its antifungal activity against Candida and is known for its disinfectant and antimicrobial property. AgNPs exhibits a higher microbial toxicity compared to other heavy metal ions. The antifungal potential of AgNP shows coherence with broad spectrum activity, multiple cellular targets and 
minimum host toxicity. However, silver is reported to be toxic at a high concentration and its persistent use causes argyrosis and argia.$^{9,10}$ Therefore, the requirement is towards exploring novel biocompatible nanomaterials with effective anti-biofilm and optical properties.

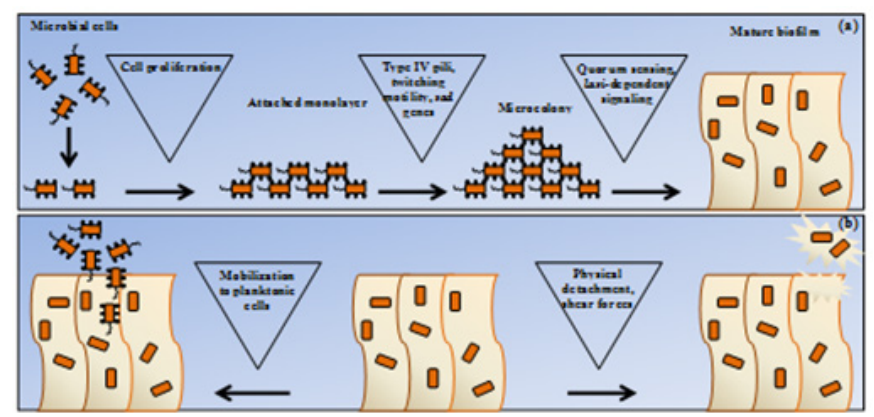

Figure I Schematic illustrations.

a. Typical example of mechanism of $P$. aeuroginisia biofilm formation. Flagella and Type IV pilli provide mobility and the biofilm matures via quorum sensing.

b. Mechanism by which biofilm detach from the adherent surface.

Semiconductor colloidal quantum dots (QDs) due to their fascinating optical properties, size-tunable emission, high quantum yield, photostability and accessibility of conjugation to functional ligands as antibodies, have made them emerge as effective agents in nanomedicines. QDs have become excellent candidates in biomedicines particularly in diagnosis, imaging, sensing and drug discovery. QDs (CdTe-MSA) conjugated with Concanavalin A (Con A) lectin have been reported to act as effective fluorescent probes for biofilm indication. ${ }^{11}$ Titania nanotubes (TNT) have also been used for studying implant associated infections and have shown to spectacularly inhibit biofilm formation on implant surfaces.$^{12}$ Various investigations also state the use of TNT based drug delivery to greatly assist in hindering implant associated infections.${ }^{13,14}$ Similarly, the use of $25 \mathrm{ppm}$ Selenium nanoparticles has been reported to inhibit almost $50 \%$ of Candida albicans biofilm, by substituting with sulfur and inducing cell structure damage. Anti-biofilm activity was reported to depend on crystallinity and size of particles. It was determined that inhibition occurred via adherence and penetration of the nanoparticles to the cells and subsequently damages the cellular structures. Therefore, crystallinity and small size allows easy conjugation and penetration in to the biofilm surface leading to better inhibition.$^{15}$ We thus envision that QDs could be considered as practical probes for investigating candidiasis infection, biofilm imaging and treatment. In spite of single drug therapy, nano-based drug combinations are likely to favour broad spectrum activity, multiple cellular targets and minimize host toxicity. It would also help address the challenge of multi drug resistance (MDR) and fungal therapeutics. Thus, by either tailoring the surface of QDs with antimicrobial drugs or entrapping them within the QD core new nano-drug formulations can be developed that would increase the potency of drugs even at a much lower concentration. Additionally, the synergetic action of the antifungal property of QDs and drugs would enhance the anti-biofilm ability . ${ }^{16}$ The use of QDs would also allow optical detection of the inhibition and favor visual monitoring of activity.$^{17}$ Furthermore, the application of low energy ion irradiation $\left(10^{12}-10^{16} \mathrm{ion} / \mathrm{cm}^{2}\right)$ can also be performed to enhance the physico-chemical properties of the QDs and in turn their antifungal potency. Literature states that both biofilm and hyphal formation are important factors that are associated with drug resistivity and virulence.$^{18}$ This invariability makes drug resistance traits and virulence traits as crucial and novel high value targets for suppressing microbial infections.

\section{Conclusion}

Thus the conception of modified QDs possessing the capability of selectively and non-lethally blocking biofilm formation and hyphae development can make them potent agents for in-vivo therapeutic utility. Further, understanding the mechanism behind QD based antifungal activity alone or in synergism with drugs would assist in developing novel therauptic strategies and develop new antifungal drug targets for MDR reversal. Hence, an interdisciplinary study involving collaborative association of synthetic chemist, physicist and biologist would help develop a "bench-to-bedside" approach with tremendous potential to expedite the discovery of new diagnostic tools with the potential of combating multidrug resistance crisis in a clinically relevant opportunistic fungal pathogen, Candida albicans and similar organisms.

\section{Acknowledgments}

This research was supported by a DST-PURSE-II funding. KR acknowledges receipt of DST-Inspire Faculty award from Department of Science and Technology, Government of India.

\section{Conflicts of interest}

None.

\section{References}

1. Li Y, Tian X Quorum Sensing and Bacterial Social Interactions in Biofilms. Sensors (Basel). 2012;12(3):2519-2538.

2. Lwin SM, Kimber I, McFadden JP Acne, quorum sensing and danger. Clin Exp Dermatol. 2014;39(2):162-167.

3. Costerton JW, Stewart PS, Greenberg EP Bacterial Biofilms: A Common Cause of Persistent Infections. Science. 1999;284(5418):1318-1322.

4. Moran G, Coleman D, Sullivan D An introduction to the medically important Candida species. In: Calderone RA \& Clancy CJ (Eds.), Candida and candidiasis. (2nd edn), ASM Press, Canada, pp. $2012 ; 387401$.

5. Chandra J, Kuhn DM, Mukherjee PK et al. Biofilm formation by the fungal pathogen Candida albicans: development, architecture, and drug resistance. J Bacteriol. 2001;183(18):5385-5394.

6. Watamoto T, Samaranayake LP, Jayatilake JA et al. Effect of filamentation and mode of growth on antifungal susceptibility of Candida albicans. Int J Antimicrob Agents. 2009;34(4):333-339.

7. Buffet Bataillon S, Tattevin P, Bonnaure Mallet M et al. Emergence of Resistance to Antibacterial Agents: The Role of Quaternary Ammonium Compounds; A Critical Review. Int J Antimicrob Agents. 2012;39(5):381-389.

8. Pinto AM, Goncalves IC, Magalhaes FD Graphene- Based Materials Biocompatibility: A Review. Colloids Surf B. 2013;111:188-202.

9. White J, Powell A, Brady K et al. Severe generalized argyria secondary to ingestion of colloidal silver protein. Clin Exp Dermatol. 2003;28(3):254-256.

10. Kwon HB, Lee JH, Lee SH et al. A case of argyria following colloidal silver ingestion. Ann Dermatol. 2009;21(3):308-310.

11. Tenorio DPL, Andrade CG, Cabral Filho PE et al. dTe Quantum Dots Conjugated To Concanavalin A as Potential Fluorescent Molecular Probes for Saccharides Detection in Candida albicans. J Photochem Photobiol. B 2015;142:237-243.

12. Lin W, Zhang $\mathrm{Y}$, Tan $\mathrm{H}$ et al. Inhibited Bacterial Adhesion and Biofilm Formation on Quaternized Chitosan-Loaded Titania Nanotubes with Various Diameters. Materials. 2016;9(3):155. 
13. Kumeria, T, Mon H, Aw MS et al. Advanced biopolymer-coated drug-releasing titania nanotubes (TNTs) implants with simultaneously enhanced osteoblast adhesion and antibacterial properties. Colloids Surf B Biointerfaces. 2015;130:255-263.

14. Mei S, Wang H, Wang W et al. Antibacterial effects and biocompatibility of titanium surfaces with graded silver incorporation in titania nanotubes. Biomaterials. 2014;35(14):4255-4265.

15. Guisbiers G, Lara HH, Mendoza-Cruz R et al. Inhibition of Candida albicans biofilm by pure selenium nanoparticles synthesized by pulsed laser ablation in liquids. Nanomedicine. 2017;13(3):1095-1103.
16. Shahverdi AR, Fakhimi A, Shahverdi HR et al. Synthesis and effect of silver nanoparticles on the antibacterial activity of different antibiotics against Staphylococcus aureus and Escherichia coli. Nanomedicine. 2007;3(2):168-171.

17. Kloepfer JA, Mielke RE, Wong MS et al. Quantum Dots as Strain- and Metabolism-Specific Microbiological Labels. Appl Environ Microbiol. 2003;69(7):4205-4213.

18. Costa-Orlandi CB, Sardi JCO, Pitangui NS et al, Fungal Biofilms and Polymicrobial Diseases. J Fungi 3(2):22. 